\section{Digitales Rathaus zwischen Angebot und Bürgernutzung}

\author{
von Martin Bechmann und Matthias Werner, \\ ITAS
}

Im Rahmen des ITAS-Projektes „Elektronische Medien und Verwaltungshandeln Demokratisierung und Rationalisierung“" wurden die Internetplattformen der Städte Karlsruhe und Mannheim als Fallstudien untersucht. Davon ausgehend, dass E-Government - neben seinen inneradministrativen Aspekten - die Außenbeziehungen des Staates neu definiert, wurden die kommunalen Internetauftritte und deren verwaltungsbezogener Kern, das Digitale Rathaus, primär als Schnittstelle von Verwaltung und Bürger begriffen. Ziel der Fallstudien war es, anhand der Zusammenführung der Angebots- (Zielsetzung und Implementierung der Stadtportale) und der Nachfrageseite (Nutzung und Nutzungspotenziale) Aufschlüsse über Akzeptanz und Perspektiven bestehender E-Government-Strategien zu erlangen. Einige Ergebnisse und Folgerungen, die sich auf die Diskrepanz zwischen dem hohen Bekanntheitsgrad der Digitalen Rathäuser und ihrer geringen Nutzung konzentrieren, werden vorgestellt.

\section{Einleitung}

Beim Blick auf die kommunalen Websites als Schnittstelle zwischen Verwaltung und Bürger drängen sich Fragen nach der Praxis der Online-Angebote, hier der Städte Karlsruhe und Mannheim, in den Vordergrund. Um hierzu die Vorstellungen und Erfahrungen der Betreiber wie auch die Resonanz der Angebote bei den Nutzern erfassen zu können, wurden im Rahmen des ITAS-Projektes „Elektronische Medien und Verwaltungshandeln - Demokratisierung und Rationalisierung "1 im Frühjahr 2002 Experteninterviews mit den Portalbetreibern, Analysen der Portale und eine telefonische Bürgerumfrage durchgeführt.

Im Folgenden werden einige Ergebnisse dieser Fallstudien vorgestellt. Dazu werden die Konzeptionen der Portalbetreiber umrissen, das konkrete Online-Angebot beschrieben und Ergebnisse der Bürgerbefragung zur Nutzung und Akzeptanz der Portale sowie Nutzungspo- tenziale vorgestellt. Anschließend werden einige Schlussfolgerungen zum derzeitigen Verhältnis von Zielvorstellungen, Implementation und Nutzung der kommunalen Internetauftritte gezogen.

\section{Die kommunalen Internetportale in Deutschland}

Die kommunalen Internetportale, die zumeist unter der Adresse www.stadtname.de zu erreichen sind, decken ein vielfältiges Angebot ab, das weit über reine Verwaltungsbelange hinaus geht (vgl. hierzu Behringer in diesem Schwerpunkt). Unter dem Begriff „Digitales Rathaus“ stellen die Stadtportale elektronische Bürgerdienste bereit ${ }^{2}$ und bilden damit im Rahmen des Gesamtkonzepts „Electronic Government“ (E-Government) einen Aspekt, der sich schnell als sehr öffentlichkeits- und medienwirksam erwiesen hat: die virtuelle Schnittstelle zwischen Verwaltung und Bürger. Dabei fungieren die Digitalen Rathäuser vornehmlich nicht als eine Ablösung, sondern als eine Erweiterung bestehender Zugangswege zur Verwaltung (Bürgerbüros bzw. Fachämter und Telefon).

Die Entwicklung der kommunalen Internetauftritte, die in ersten Kommunen ab Mitte der 1990er Jahre einsetzte, kann mit der Formel „vom Stadtinformationssystem zum Portal“" (Stapel-Schulz, Eifert, Siegfried 2002，S. 5) charakterisiert werden. Damit wird zum einen die Erweiterung der Inhalte der Portale beschrieben, die vermehrt dem Anspruch folgte, das Stadtleben möglichst umfassend virtuell abzubilden. So wurde z. B. damit begonnen, die Stadtportale auch zur Einbindung der Bürger in kommunale Politikprozesse zu nutzen, Vereinen und Initiativen Raum zur Selbstdarstellung zu bieten oder $-\mathrm{z}$. T. mit privaten Kooperationspartnern - auch die lokale Wirtschaft über virtuelle Marktplätze in die Portale $\mathrm{zu}$ integrieren. Zum anderen drückt diese Formel aus, dass die zunächst rein auf Auskünfte (z. B. Ämteröffnungszeiten und Zuständigkeiten, kulturelle und touristische Informationen) beschränkten Stadtinformationssysteme im Sinne eines Ausbaus in die Tiefe schrittweise um interaktive Anwendungen ergänzt wurden. Zur Vorbereitung von Behördengängen wurden zudem Formularserver eingerichtet und 
schließlich einzelne transaktive Dienste zur Ersetzung des Ämtergangs installiert. ${ }^{3}$

\section{Die Fallbeispiele Karlsruhe und Mann- heim}

Im Folgenden sollen wesentliche Kennzeichen der Konzeptionen der Stadtportale von Karlsruhe und Mannheim aufgezeigt werden, um prüfen zu können, inwiefern diese sich auf die konkreten Realisierungen und die Nutzung auswirken. ${ }^{4}$

\subsection{Die Konzeptionen der Stadtportale}

\section{Ideen und Erwartungen}

Beide Städte starteten ihre Internetauftritte 1995/96, die Portale dienten zunächst primär der Information über das Stadtgeschehen und Belangen der Stadtverwaltung. Die Informationsund Serviceangebote der Portale wurden - ana$\log$ der beschriebenen allgemeinen Entwicklung - schrittweise erweitert und zunehmend durch interaktive Anwendungen und die Digitalen Rathäuser mit ihren Online-Verwaltungsdienstleistungen ergänzt.

Die Konzepte beider Städte fühlen sich dem Leitbild der Kunden- bzw. Bürgerorientierung verpflichtet, indem sie versuchen, ihren Webauftritt angepasst an die Ausgangssituation und Bedürfnislage der Bürger zu konzipieren und zu realisieren, sie weisen aber in der Ausgestaltung Unterschiede auf.

Der Karlsruher Ansatz stützt sich auf die Erfahrung, dass die Websites der Städte mit gezielten Anliegen aufgesucht werden, und konzentriert sich daher auf die Sicherung eines „hohen Gebrauchswerts" des Angebots: Die Nutzer sollen auf einfache und schnelle Weise zu den gesuchten Inhalten gelangen, die möglichst umfassend und aktuell zu halten sind. Damit bleibt das Karlsruher Konzept, das von der Stadtverwaltung in Eigenregie betrieben wird, weitgehend auf stadt(verwaltungs)bezogene Informationen und Dienste konzentriert, was zu einer stärkeren Abbildung der Verwaltungsstrukturen führt. Auf die Integration eines Marktplatzbereichs und die Kooperation mit privatwirtschaft- lichen Partnern wird bewusst verzichtet (vgl. Behringer).

Mannheims Ansatz zur Ausgestaltung der Idee der Bürgerorientierung setzt einen anderen Schwerpunkt. Hier wird zusätzlich zum Angebot des Digitalen Rathauses versucht, die Bürger durch eine an „Lebenslagen“ ${ }^{\text {“5 }}$ ausgerichtete Nutzerführung zu den Verwaltungsangeboten zu führen. Der Mannheimer Netzauftritt ist zudem dadurch gekennzeichnet, dass er das Angebot der öffentlichen Hand mit Präsentationsmöglichkeiten für lokale Unternehmen verknüpft. Organisatorisch drückt sich die stärkere Einbeziehung der lokalen Wirtschaft in das Angebot des Stadtportals darin aus, dass der „virtuelle Marktplatz" auf Mannheim.de in Public-Privat-Partnership betrieben wird. Mannheim.de löst sich damit stärker als Karlsruhe.de von bestehenden Verwaltungsstrukturen und öffnet sich weiteren Akteuren. ${ }^{6}$

Das Digitale Rathaus selbst wird in der Konzeption beider Städte als Weiterführung der Bürgeramtsidee im virtuellen Raum verstanden. D. h. es soll im Internet ein Ort etabliert werden (,One-Stop-Government"), an dem die Verwaltung den Bürgern ihre Dienstleistungen nicht nach Ämterstrukturen und Zuständigkeiten, sondern ausgehend von den Nutzeranliegen zentral gebündelt anbietet. In den Digitalen Rathäusern geschieht dies insbesondere dadurch, dass hier keine Trennung nach Fachämtern erfolgt, sondern die elektronischen Verwaltungsdienste themenorientiert (Meldewesen, KfzAngelegenheiten, Abfall, ...) auf gemeinsamen Seiten verfügbar sind. Übereinstimmend sehen die Vertreter beider Städte das Primärziel des Digitalen Rathauses in der Vorbereitung und Erleichterung von Behördengängen, während es (aktuell) erst an zweiter Stelle darum gehen könne, Behördengänge zu ersetzen.

\section{Stand der Umsetzung: Kurzportraits von Karls-} ruhe.de und Mannheim.de

Auf den Startseiten ihrer Internet-Auftritte präsentieren sich Karlsruhe und Mannheim? ${ }^{7}$ abgesehen von gestalterischen Aspekten, mit einem ähnlichen Angebot: Empfangen werden die Nutzer mit aktuellen Nachrichten aus dem Stadtgeschehen sowie mit Service-Angeboten wie Veranstaltungshinweisen, Stadtplan oder 
dem E-Mail-Abonnement städtischer Pressemitteilungen.

Die weitere Nutzerführung unterscheidet sich allerdings deutlich. Karlsruhe.de erschließt sich dem Nutzer über eine Überblicksseite, die unter mehreren Themenblöcken wie „Stadtinformation und Tourismus“, „Politik und Verwaltung“ oder „Wirtschaft" auf weitere Angebote verweist. Die über diese Themenblöcke erreichbaren Seiten unterliegen dabei keiner durchgängig einheitlichen Struktur und keinem einheitlichen Erscheinungsbild. Zum „Digitalen Rathaus", auf das auf der Startseite nicht direkt verwiesen wird, findet man auf der Überblicksseite nicht über diesen Begriff, sondern über den Link „Elektronische Dienste und Vordrucke“. Mannheim.de bietet dagegen eine Navigation mit einheitlichem Webdesign, was nicht nur graphisch ansprechender und ,ambitionierter“" erscheinen mag, sondern die Bewegung auf den Seiten durch gleichartige Navigationsleisten erleichtert. Über eine feste Navigationsleiste besteht die Auswahl von Angeboten aus den Bereichen „Bürger und Stadt", „Tourismus“, „Marktplatz“ und „Lebenslagen“, während eine weitere Menüleiste Wahlmöglichkeiten aus den jeweils gewählten Bereichen bereit hält. Auf der Startseite sind über diese Menüleiste die Dienste des „Digitalen Rathauses“ mit einem Mausklick zu erreichen. ${ }^{8}$

\subsection{Information - Interaktion - Transaktion}

Zur systematischen Erfassung der Angebote der Stadtportale bzw. Digitalen Rathäuser wurden in den letzten Jahren verschiedene Schemata zugrunde gelegt, deren Klassifizierungen der Anwendungsfelder von Online-Angeboten zumeist ähnlich angelegt sind. ${ }^{9}$ Das hier herangezogene dreigliedrige Schema Information - Interaktion - Transaktion ${ }^{10}$ umfasst unter seinen Grundbegriffen folgende Anwendungsarten:

- Information: reine Bereitstellung von Auskünften und Nachrichten zu Verwaltungsangelegenheiten, Kommunalpolitik, lokalen Unternehmen und dem Stadtgeschehen im Allgemeinen

- Interaktion: Kommunikationsmöglichkeiten mit Verwaltung und Gemeinderat bzw. für die Bürger untereinander (E-Mail, Chats, Foren), Formular-Bestellung oder -Download
- Transaktion: Online-Veranlassung und Abwicklung von Dienstleistungen, insbesondere von elektronischen Verwaltungsakten. ${ }^{11}$

Die folgende Darstellung beschränkt sich im Wesentlichen auf Angebote aus den Bereichen elektronische Verwaltung (E-Administration) und elektronische Demokratie (E-Democracy).

\section{Information}

Die konzeptionellen Unterschiede finden in den inhaltlichen Schwerpunktsetzungen der Stadtportale Ausdruck. Karlsruhe.de stellt sich insbesondere als Informationsdienst der Stadtverwaltung dar. Neben Informationen zu Verwaltungsdiensten (Öffnungszeiten, Zuständigkeiten, benötigte Dokumente) und städtischen Einrichtungen nutzt Karlsruhe sein Portal insbesondere auch, um über die laufenden Bürgerbeteiligungsverfahren zu informieren. Dabei bleibt es aber hauptsächlich beim Informationsaspekt, d. h. es wird online für offline-Prozesse geworben. Zudem wird an mehreren Stellen auf Möglichkeiten zu bürgerschaftlichem Engagement in der Stadt hingewiesen. Das Angebot zur Information über die Aktivitäten des Gemeinderats ist hingegen nur schwach ausgeprägt.

Während auf Mannheim.de dem Aspekt von Bürgerbeteiligung und -engagement keine herausgehobene Rolle zukommt, kommt ein anderer Aspekt von E-Democracy zum Tragen, indem - dem Gedanken des Demokratie fördernden Effekts von Transparenz folgend - auf den Seiten des Gemeinderats nicht nur Informationen über seine Zusammensetzung sowie die Seiten der Mitglieder und Fraktionen (oder Links zu diesen) zu finden sind, sondern auch die Haushaltsberatungen dokumentiert sind und die Ratsvorlagen für die Gemeinderatssitzungen veröffentlicht werden. Neben dem Bereich „Bürger \& Stadt", der ähnliche Inhalte wie die entsprechenden Karlsruher Seiten bietet, bemüht sich Mannheim.de um die Verknüpfung von Informationen zu öffentlichen und privatwirtschaftlichen Angeboten.

\section{Interaktion}

Kommunikationsmöglichkeiten mit den Stadtverwaltungen sind in beiden Städten durch Angaben der E-Mail-Adressen der für die je 
weiligen Dienstleistungen zuständigen Ämter gegeben wie auch die der konkreten Ansprechpartner, allerdings nicht durchgängig. Ebenso sind in der Regel Telefonnummern und Dienstzeiten angegeben, vereinzelt wird auch um Rückmeldungen über die Zufriedenheit mit dem Internetangebot und dem Service der jeweiligen Verwaltungseinheit gebeten (per E-Mail, HTML-Formular oder Briefpost).

Sofern die im Internet verfügbaren Formulare für Verwaltungsdienste (vgl. Tab. 1) wegen der festgeschriebenen Notwendigkeit der Unterschrift nicht online an die zuständige Stelle versendet werden können, besteht zumeist die Möglichkeit zum Download der For- mulare, die ausgefüllt per Post verschickt ${ }^{12}$ oder zum Ämterbesuch mitgebracht werden können. Hilfsangebote für das Ausfüllen der Formulare beschränken sich, wenn überhaupt vorhanden, auf knappe Erläuterungen oder die Möglichkeit zum Download von Informationsblättern. Für weitere Hilfestellungen zu Verwaltungsangelegenheiten sind auf beiden Portalen Telefonnummern angegeben.

Möglichkeiten zur Kommunikation mit den Gemeinderatsmitgliedern via E-Mail sind für Karlsruhe wie auch für Mannheim für die Mehrzahl der Ratsmitglieder gegeben. Eine Homepage oder nur eine persönliche E-MailAdresse des Bürgermeisters sucht man hinge

Tab. 1: Online-Dienstleistungen der Digitalen Rathäuser auf Karlsruhe.de und Mannheim.de (Stand: Frühjahr 2002)

\begin{tabular}{|c|c|c|}
\hline & KARLSRUHE & MANNHEIM \\
\hline 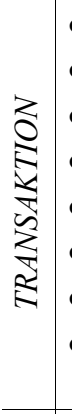 & $\begin{array}{ll}\text { - } & \text { Auskunft aus dem Melderegister } \\
\text { - } & \text { Kfz-Wunschkennzeichen } \\
\text { - } & \text { Widerspruch zur Übermittlung von Daten } \\
\text { - } & \text { Bestellung standesamtlicher Urkunden } \\
\text { - } & \text { Meldepflicht der Wohnungsgeber } \\
\text { - } & \text { Bestellung, Änderung von Abfalltonnen } \\
\text { - } & \text { Verzicht auf die Biotonne }\end{array}$ & 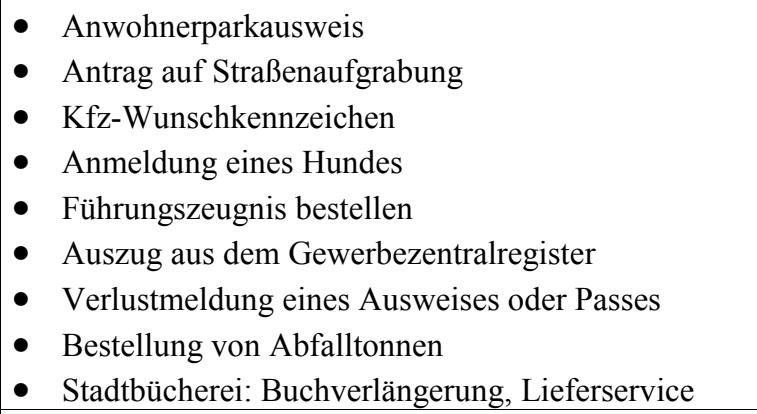 \\
\hline $\begin{array}{l}z \\
0 \\
\vdots \\
\vdots \\
z \\
\mathbb{z} \\
\vdots \\
\vdots\end{array}$ & $\begin{array}{l}\text { - Abmeldung von Karlsruhe** } \\
\text { - } \text { Anmeldung in Karlsruhe, Ummeldung innerhalb } \\
\text { - Antsruhes*** } \\
\text { - } \text { Gewerbeanmeldung, -abmeldung, -ummeldung** } \\
\text { - } \text { Ausstellung, Änderung, Ersatz von Lohnsteuerkar- } \\
\text { ten** } \\
\text { - Ausstellung, Verlängerung der Aufenthaltserlaub- } \\
\text { nis/Aufenthaltsgenehmigung*** } \\
\text { - Fischereischeine*** } \\
\text { - Anmeldung einer Lebenspartnerschaft*** } \\
\text { - Erteilung, Verlängerung, Erweiterung einer Reise- } \\
\text { gewerbekarte*** } \\
\text { - Feststellung einer Behinderung und Ausstellung des } \\
\text { Ausweises*** } \\
\text { Erhöhung des Grades einer Behinderung und Ver- } \\
\text { längerung des Ausweises*** }\end{array}$ & $\begin{array}{l}\text { - } \text { Ausweisanträge* } \\
\text { - } \text { Ummeldung innerhalb Mannheims* } \\
\text { - } \text { Förderung des Ersteinbaus von Bad/Dusche sowie } \\
\text { - } \text { Außnahmen zur Energieeinsparung** } \\
\text { ten** } \\
\text { - Widerspruch zur Übermittlung von Daten** } \\
\text { - Bestellung standesamtlicher Urkunden** } \\
\text { - Befreiung von der Ausweispflicht** } \\
\text { - Meldepflicht des Wohnungsgebers** } \\
\text { - Ausstellung, Verlängerung der Aufenthaltserlaub- } \\
\text { - nis/Aufenthaltsgenehmigung*** } \\
\text { Suche von Fundsachen des Fundbüros }\end{array}$ \\
\hline * & \multicolumn{2}{|c|}{$\begin{array}{l}\text { Online-Ausfüllen des Formulars und Online- oder Postversand, später persönliche Unterschrift bei der zu- } \\
\text { ständigen Verwaltungsdienststelle }\end{array}$} \\
\hline $\begin{array}{l}* * \\
* * *\end{array}$ & \multicolumn{2}{|c|}{$\begin{array}{l}\text { Download und Ausdruck des Formulars und persönliche (oder bevollmächtigte) Vorlage des unterschriebe- } \\
\text { nen Formulars }\end{array}$} \\
\hline
\end{tabular}


gen in beiden Stadtportalen vergebens.

In Karlsruhe wurde mit dem Bürgerbeteiligungsverfahren „City 2015“, in dem es um die zukünftige Gestaltung der Innenstadt ging, mit einer über rein das Verfahren bewerbende Aspekte hinaus gehenden Nutzung des Internets experimentiert. Mit der Seite http://www. city2015.de wurde eine Plattform geschaffen, die das Verfahren online begleitete, indem unter anderem Entwürfe von Stadtplanern präsentiert, die Ergebnisse der verschiedenen Arbeitsgruppen dokumentiert und zum Informationsaustausch und Einbringen von Ideen in den offlineProzess ein Forum eingerichtet wurde. ${ }^{13}$

\section{Transaktion}

Während die Informations- und Interaktionsangebote zu Verwaltungsdiensten in erster Linie dem Ziel dienen, Behördengänge zu erleichtern und vorzubereiten, sollen die transaktiven Dienste die Behördengänge selbst ersetzen, indem durch das Ausfüllen und Versenden eines Formulars die entsprechende Verwaltungsdienstleistung veranlasst wird. Beide Städte haben bereits Ende der 1990er Jahre damit begonnen, einzelne Verwaltungsdienste mit transaktiven Elementen anzubieten. Die online ohne Medienbruch gegebenen Möglichkeiten zu Transaktionen mit der Verwaltung sind aber aufgrund bestehender Rechtsvorschriften (vor allem Schriftformbindung) und technischer wie organisatorischer Hemmnisse gering. Beide Städte bemühen sich, Anwendungen zur Verfügung zu stellen, doch sind die bisher für die Bürger nutzbaren Angebote nur bedingt von Relevanz (z. B. Reservierung des Kfz-Wunschkennzeichens oder Anmeldung eines Hundes). Das vorhandene Angebot beider Städte ist ähnlich in Auswahl und Umfang (vgl. Tab. 1).

Der Nutzwert eines Angebots mit Transaktionsmöglichkeit ist gegenüber dem interaktiven Angebot „Formular-Download mit Medienbruch" zwar dadurch, dass der Bürger sein Anliegen komplett online durchführen kann, deutlich höher. Betrachtet man aber die unter den beschriebenen Restriktionen verwirklichten transaktiven Dienste, so kann die Bewertung des Nutzens von Transaktionsangeboten sich nicht auf das Kriterium „medienbruchfreie Abwicklung“ beschränken. Die bisher realisierten Online-Services scheinen in zweifacher
Hinsicht wenig bedarfsorientiert, was ihren Nutzwert für die Bürger wie auch für die Verwaltung, und damit die Fallzahlen ihrer Nutzung, einschränkt und den für ihre Bereitstellung betriebenen Aufwand in Frage stellen kann: Die Angebote treffen zum einen sicher nicht den Bedarf einer breiten Masse (z. B. Angebote für Hundehalter), zum anderen werden sie bei den einzelnen Nutzern selten benötigt (wie oft meldet man einen Hund an, bestellt man ein Kfz-Wunschkennzeichen?).

\subsection{Rationalisierung-Demokratisierung}

Die Verortung der auf Karlsruhe.de und Mannheim.de realisierten Angebote in den Dimensionen Rationalisierung und Demokratisierung entspricht - unabhängig von den unterschiedlichen Schwerpunktsetzungen der Portalkonzepte - in der Tendenz den Ergebnissen der „eTown 2002“-Studie. Diese zeigte auf, dass in den kommunalen Umsetzungen von E-Government Aspekte elektronischer Demokratie gegenüber denen der elektronischen Verwaltung bislang deutlich weniger Aufmerksamkeit gefunden haben. In der Gesamtbewertung der Portale durch die Studie wie auch in den Einzelbewertungen hinsichtlich der Qualität ihres Angebots in den Bereichen E-Administration und E-Democracy befinden sich die beiden Städte jeweils in der Spitzengruppe deutscher E-Government-Umsetzungen (Initiative D21 2002a, S. 3 u. S. 16 ff.).

Der Vergleich der beiden Städte in Bezug auf die Rationalisierungsdimension von E-Government an der Schnittstelle zu den Bürgern ergibt folgende Befunde:

- Die Angebote zur Information über Verwaltungsbelange, also im Kern zur Vorbereitung und Erleichterung von Behördengängen, sind bei beiden Städten am weitesten und recht umfassend ausgebaut. Auch das verfügbare Angebot an tiefer greifenden Anwendungen für die Bürger (Interaktion und Transaktion) ist ähnlich. Die Unterschiede in den Konzeptionen der Stadtportale schlagen hier nur in geringem Maß auf die Angebote der Digitalen Rathäuser durch. ${ }^{14}$

- Die Digitalen Rathäuser sind zunächst als ein zu den Zugangswegen Bürgeramt und Telefon komplementäres Angebot zu verstehen, 
dessen Bereitstellung zusätzliche Kosten verursacht. Beim aktuellen Umsetzungsstand kann das Digitale Rathaus nur in Ausnahmen den Gang zu den Bürger- oder Fachämtern ersetzen. Ein Nutzen für die Bürger (und Verwaltung) kann aber bereits in der Möglichkeit des Formular-Downloads und der möglichst vollständigen Vorbereitung eines Behördengangs gesehen werden (vgl. Behringer; Lenk 2002, S. 5).

Insgesamt findet die Rationalisierungsdimension zurzeit stärker in Form von Effektivitätssteigerungen im Kontakt mit den Bürgern Ausdruck denn als Einsparungen und Rationalisierungsgewinnen. Zunächst bedeutet die Bereitstellung von transaktiven Diensten einen Mehraufwand, der bisher nicht zu einem realisierten Angebot führte, das auf einen größeren Bedarf trifft und insofern geeignet wäre, nennenswerte Fallzahlen zu erreichen. Einsparungen durch die elektronische Abwicklung von Verwaltungsdienstleistungen sind bestenfalls langfristig eine realistische Perspektive.

Als Resultate bezüglich der Demokratisierungsdimension von Stadtportalen und Digitalen Rathäusern können aus den Fallstudien genannt werden:

- Noch stärker als die Rationalisierungsdimension bleibt die Demokratisierungsdimension im Wesentlichen auf die Bereitstellung von Informationen aus der Verwaltung und dem Rat beschränkt. Das (besonders in Karlsruhe) nur mäßig ausgeprägte Informationsangebot zur Arbeit des Gemeinderats weist darauf hin, dass die durch das Internet gegebene technische Möglichkeit zur Erhöhung der Transparenz von (Kommunal-) Politik auf die Bereitschaft zu ihrer Nutzung und die Kooperation von Rat und Verwaltung treffen muss.

- Die Nutzung des Internets zur institutionalisierten Einbindung der Bürger in lokale Entscheidungsprozesse beschränkt sich - abgesehen vom Karlsruher Beteiligungsverfahren „City 2015“, das auf einer eigenen Website interaktive Inhalte bot - auf die Bewerbung und Dokumentation von partizipativen ,offline-Projekten" sowie die Möglichkeit, sich per E-Mail an Ansprechpartner aus dem Rat oder der Verwaltung zu wenden.
Die Demokratisierungsdimension von E-Government zeigt sich somit in den Umsetzungen beider Städte nur wenig ausgeprägt. Am ehesten können Schritte in Richtung einer E-Democracy auf der kommunalen Ebene in Ansätzen zur Herstellung von Transparenz der Kommunalpolitik und Verwaltung gesehen werden.

\section{Bürgerbefragung: Wissen, Nutzung und Akzeptanz von kommunalen Online- Angeboten}

Im Februar 2002 wurden im Rahmen des Projektes in den Städten Mannheim und Karlsruhe jeweils rund 500 Telefoninterviews durchgeführt. Befragt wurden Einwohner im Alter über 16 Jahren, die in den letzten zwei Jahren mit städtischen Ämtern und Behörden zu tun hatten. ${ }^{15}$ Die Bürger wurden zu Computer- und Internetnutzung, Bekanntheit und Nutzung der Stadtportale, potenziellen E-Government-Angeboten und $\mathrm{zu}$ den städtischen Bürgerämtern befragt.

Im Folgenden werden einige Ergebnisse dieser Befragung vorgestellt. Im Mittelpunkt stehen dabei die städtischen Internet-Portale in ihrer Funktion als Schnittstelle zwischen dem Bürger einerseits und kommunaler Verwaltung und Politik andererseits. Es geht hier also in erster Linie um den engeren Bereich des „Digitalen Rathauses" und den mit dem E-Democracy-Konzept zusammenhängenden Angeboten, weniger um andere Funktionen der Stadtportale wie Imagepflege, Stadtmarketing oder Förderung der lokalen Wirtschaft.

Eine Untersuchung der Stadtportale als Schnittstelle zwischen Verwaltung und Bürger aus Bürgersicht muss Fragen stellen nach der Zugänglichkeit und Wahrnehmung der Angebote und der Akzeptanz bzw. dem Wunsch nach einem Ausbau derselben seitens der Bürger. Zur Beantwortung dieser Fragen wird zunächst auf die Zugangsmöglichkeiten zu Online-Angeboten eingegangen: Wer hat überhaupt Zugang zum Internet und somit die Möglichkeit, entsprechende Angebote zu nutzen? Wo liegen hier die Zugangshürden? Daraufhin wird die konkrete Wahrnehmung der bestehenden Angebote durch die Bürger untersucht, um zum Schluss einige Befunde zu potenziellen Angeboten vorzustellen. 


\subsection{Computer- und Internetnutzung}

Die Befragung zeigte, dass in beiden Städten mit annähernd $75 \%$ ein großer Anteil der Bürger einen Computer nutzt. Fast zwei Drittel der Befragten haben auch Zugang zum Internet, wobei hier nicht nach dem Ort der Nutzung (von $\mathrm{zu}$ Hause, von der Arbeit, von anderen Orten aus) unterschieden wurde. $53 \%$ der Befragten haben von zu Hause aus Zugang zum Internet.

Internet- und Computernutzung sind, wie aus zahlreichen Studien (vgl. z. B. Initiative D21 2002b) bekannt, sozialstrukturell ungleich verteilt. Dies wird für gewöhnlich als „Digital Divide“ oder digitale Spaltung bezeichnet, und meint sowohl unterschiedliche Zugangschancen zu den neuen Medien als auch sich daraus ergebende Verstärkungen sozialer Chancenungleichheiten. ${ }^{16}$

Auch in Karlsruhe und Mannheim zeigt sich das bekannte Bild: Der Internetzugang ist ungleich nach Alter, Bildungsgrad, Erwerbstätigkeit und Geschlecht verteilt (vgl. hierzu auch Behringer, Tab. 1 auf S. 59). Ausländer, insbesondere Immigranten, sind insgesamt seltener online als Deutsche.

Betrachtet man die Gründe der Nichtnutzung von Computern (vgl. Abb. 1), zeigt sich, dass bei der maßgeblichen Kategorie der Nichtnutzer der wichtigste Grund die Unsicherheit im Umgang mit der Technik ist, gefolgt von einer generellen, kategorischen Ablehnung (,Ich will grundsätzlich nichts damit $\mathrm{zu}$ tun haben"). Auch Kostengründe und eine fehlende Notwendigkeit der Verwendung von Computern oder Internet im Alltag spielen für jeweils rund ein Fünftel der Nichtnutzer eine Rolle, haben aber weniger Bedeutung im Vergleich zu den Unsicherheiten im Umgang mit der Technik und kategorischer Ablehnung. Bei denjenigen, die einen Computer verwenden, aber keinen Zugang zum Internet haben, geben zwei Drittel an, das Internet im Alltag nicht zu brauchen, an zweiter Stelle steht mit noch rund einem Drittel der „Offliner“ der Kostenfaktor.

Was verbirgt sich nun hinter einer kategorischen Ablehnung von Computern und Internet? Die sozialstrukturelle Analyse der „Totalverweigerer" zeigt, dass sich diese Gruppe hauptsächlich aus denjenigen zusammensetzt, die entsprechend der Trennlinien des Digital Divide am stärksten benachteiligt sind. Dieje- nigen, die statistisch die größten Hindernisse zur Nutzung der neuen Medien überbrücken müssen, lehnen die entsprechende Technik am ehesten kategorisch ab.

\section{Abb. 1: Gründe für die Nichtnutzung von Computer und Internet}

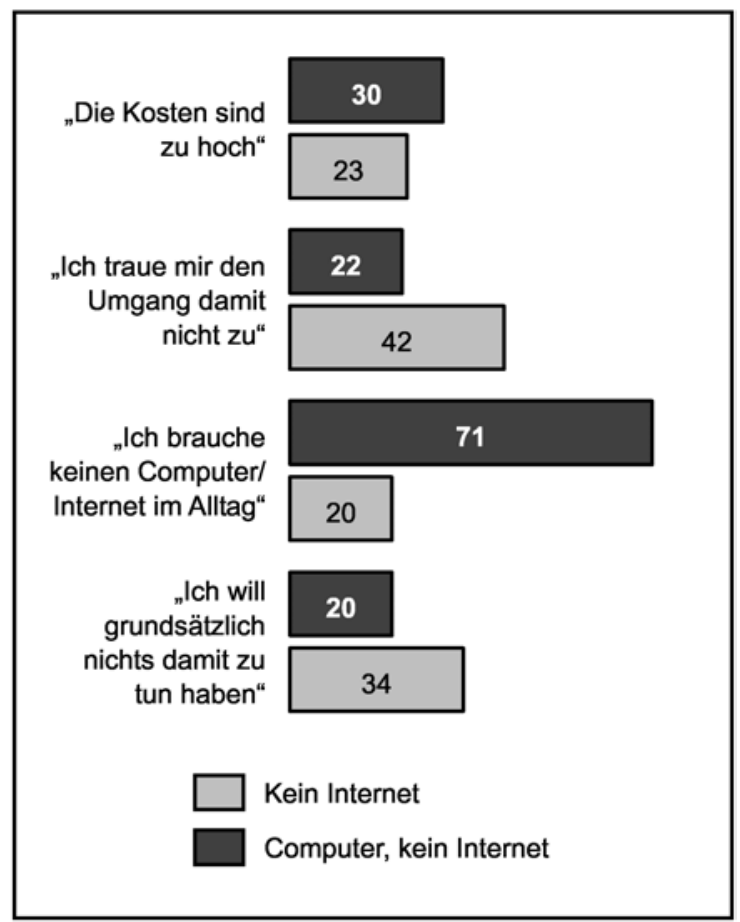

Weiterhin ist vor dem Hintergrund des kommunalpolitischen Bezuges von städtischen Internetportalen von Interesse, ob es einen $\mathrm{Zu}$ sammenhang zwischen Internet- und Computernutzung und Politikinteresse, insbesondere bei kommunalpolitischen Themen, gibt. Dabei zeigt sich, dass zwar das Interesse für Politik tendenziell mit der Nutzung der neuen Medien korreliert, dass aber diejenigen, die sich regelmäßig über kommunalpolitische Themen informieren, seltener als andere das Internet nutzen (vgl. Abb. 2). Dies hängt zum großen Teil mit dem Faktor Alter zusammen: Mit zunehmenden Alter werden in der Regel kommunalpolitische Themen wichtiger, z. B. durch Hausund Grundbesitz (vgl. Holtkamp in diesem Schwerpunkt). Gerade Ältere nutzen jedoch seltener Computer und Internet.

Diese Befunde bestätigen die in den Stuttgarter Bürgerforen (vgl. Fuchs/Kastenholz in diesem Schwerpunkt) geäußerten Befürchtun 
gen einer sozialen Spaltung in Informierte und Uninformierte. Besonders betroffen sind aus dieser Perspektive sozial Schwache und ältere Menschen.

\section{Abb. 2: Techniknutzung und Politikinteresse}

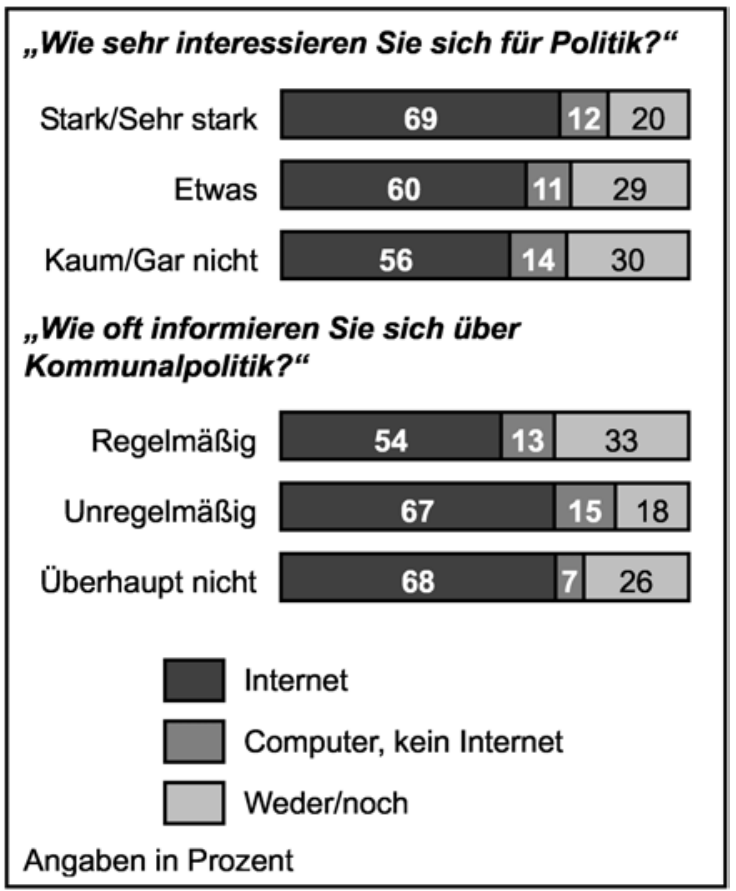

\subsection{Nutzung der städtischen Angebote im Internet}

Hinsichtlich der Internetportale der untersuchten Städte wird, wie bereits beschrieben, zwischen dem Portal im Allgemeinen, welches unterschiedlichste, allgemeine Informationen bietet, und dem Bereich des Digitalen Rathauses unterschieden, in dem sich die von den Städten angebotenen Online-Verwaltungsdienstleistungen finden,. Beide Angebotsbereiche wurden gesondert untersucht.

\section{Stadtportale}

Zwei Drittel derer, die Zugang zum Internet haben, hatten bereits das Stadtportal gesehen. Informative Angebote der Stadtportale, wie z. B. Informationen über die Stadt und städtische Nachrichten, werden bevorzugt angesteuert. Mehr als die Hälfte derjenigen, die die Portale nutzen, tun dies, um sich über die Angebote der
Verwaltung zu informieren. Nur acht Prozent der Portalbesucher hingegen waren an der Arbeit des Gemeinderates interessiert.

Die Portale werden von den Besuchern insgesamt sehr positiv bewertet (vgl. Abb. 3). Während das Mannheimer Portal insbesondere im Hinblick auf die Benutzerfreundlichkeit (Übersichtlichkeit, Orientierung, Hilfen) von rund $80 \%$ der Nutzer als gut bewertet wird, bekommt das Karlsruher Portal höhere Zustimmungswerte bei den Inhalten (Aktualität und Nützlichkeit). Alles in allem entsprechen die Unterschiede in der Bewertung beider Portale zwar den unterschiedlichen zugrunde liegenden Konzeptionen (s. o.), sind in der Größenordnung aber gering.

\section{Abb. 3: Bewertung der Stadtportale}

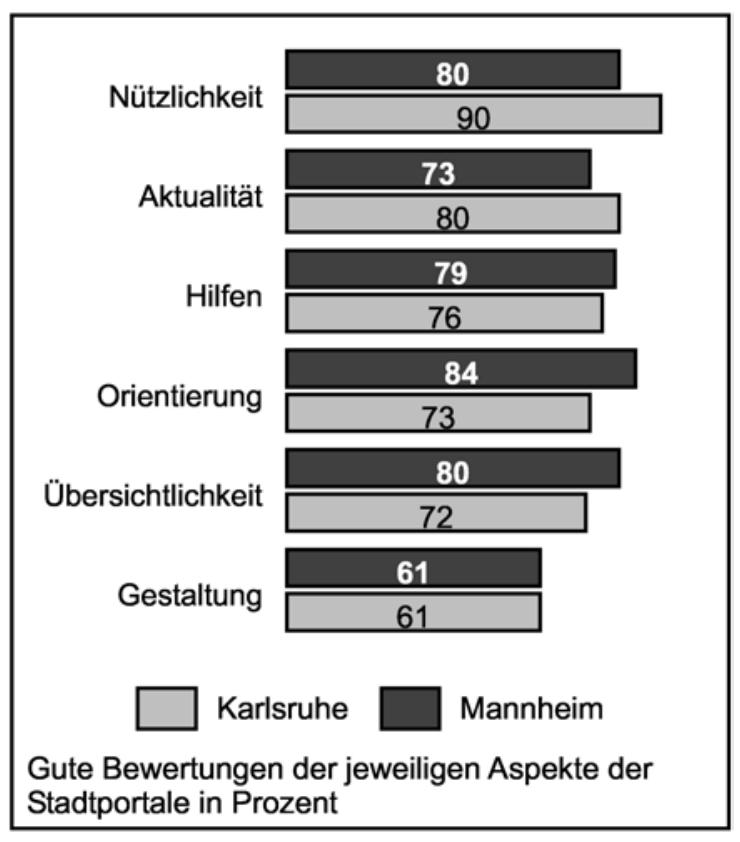

\section{Digitales Rathaus}

Trotz der großen Bekanntheit der Stadtportale werden die originären Verwaltungsdienstleistungen des Digitalen Rathauses erstaunlich selten in Anspruch genommen. Zwar gaben immerhin $35 \%$ aller Befragten an, ihnen sei die Möglichkeit, Verwaltungsdienste per Internet abzuwickeln, bekannt, doch lediglich 24 von insgesamt 1005 Befragten gaben an, dass sie diese Möglichkeit bereits genutzt hätten. Diese Gruppe lässt sich aufgrund der geringen Fallzahl leider kaum weiter differenzieren, 
doch teilt der Großteil der Gruppe einige Merkmale (Mehrfachantworten möglich):

- Diese Nutzer haben von zu Hause (22 Nutzer) oder vom Arbeitsplatz (18) Zugang zum Netz, in der Regel somit sogar von beiden Orten (17).

- Sie sind fast alle voll beschäftigt (20) und haben Angestelltenstatus (18) mit gehobener Tätigkeit (12).

Auch bei der Nutzung des Internets für Verwaltungsangelegenheiten durch diese kleine Gruppe stand die Informationsbeschaffung im Vordergrund: Am häufigsten wurde sich darüber informiert, welches Amt für bestimmte Angelegenheiten zuständig ist (18) oder welche Unterlagen benötigt werden (14). Erst danach kommen Formularbestellungen oder Downloads (jeweils 9 Nennungen). Die Möglichkeiten, Transaktionen gleich per Internet zu erledigen, wurden am seltensten in Anspruch genommen: Nur siebenmal wurde ein Formular online an ein Amt geschickt.

Es zeigt sich also, dass, insoweit die Dienste des Digitalen Rathauses in Anspruch genommen werden, dies hauptsächlich vorbereitend $\mathrm{zu}$ einem Amtsgang geschieht. Dies ist verständlich, wenn man bedenkt, dass insbesondere die Möglichkeiten der Transaktion per Internet, also die Einreichung von Formularen per Webinterface oder E-Mail, noch relativ gering sind und auch herkömmliche Arten der Kommunikation nicht vollständig ablösen oder den Gang auf das Rathaus bzw. ins Bürgerbüro ersparen können. Den Angeboten des Digitalen Rathauses mangelt es in dieser Hinsicht noch an Tiefe und Breite, d. h. einerseits ist es nur in wenigen Fällen möglich, eine einen Behördengang ersparende Transaktion vollständig über das Internet abzuwickeln, andererseits betreffen die Angebote an Transaktionen, die jetzt schon vorhanden sind, oftmals nur einen Teil der Bürger und machen nur einen kleinen Teil der Behörden-Bürger-Transaktionen aus. Der Nutzwert der Angebote des Digitalen Rathauses für den Bürger hängt jedoch genau von diesen Punkten $a b, d . h$. erst wenn das Internet den Gang zum Rathaus erspart, ist ein deutlicher Nutzen vorhanden - und je öfter bestimmte Angebote in Anspruch genommen werden, desto höher ist der Nutzwert. In Zusammenhang mit der feststellbaren hohen Gebrauchs- wertorientierung der Portalbesucher (vgl. Behringer) bietet sich so der noch eingeschränkte Nutzwert des Digitalen Rathauses als ein wichtiger Grund für die geringe Inanspruchnahme der Dienste an.

Festzuhalten ist zunächst, dass hauptsächlich Bürger, die aufgrund ihrer Internetpraxis von zu Hause als auch vom Arbeitsplatz aus gut mit dem Medium vertraut sind, den Weg ins Digitale Rathaus finden. Ein sicherer Umgang mit den Möglichkeiten der Technik ist also - über die einfache Computer- und Internetbedienung hinaus - Voraussetzung für die Inanspruchnahme städtischer Online-Angebote. Aber auch von dieser versierten Gruppe der Internetnutzer nimmt nur ein kleiner Teil die Angebote des Digitalen Rathauses wahr.

\subsection{Nutzungspotenziale}

Bei der Betrachtung von E-GovernmentAngeboten war über den augenblicklichen Stand der Implementation hinaus auch die Akzeptanz potenzieller Angebote von Interesse. Während bei der Betrachtung bestehender Angebote Online-Verwaltungsdienstleistungen im Vordergrund standen, wurden bei der Potenzialanalyse auch weitergehende, auf politische Partizipation zielende Angebote untersucht, wie z. B. die Möglichkeit, im Internet zu wählen oder kommunalpolitische Diskussionsforen zu aktuellen Themen einzurichten.

Zunächst wurde jedoch danach gefragt, ob die Bürger es begrüßen würden, wenn das Internet alle Ämtergänge überflüssig machen würde. Darauf antworteten $70 \%$ aller Befragten mit „Ja“. Dabei verteilte sich die Zustimmung zu dieser Frage wiederum entlang der sozialräumlichen Linien des „Digital Divide“: Je höher das Bildungsniveau und die berufliche Stellung, je geringer das Alter, desto größer war der Wunsch nach einer Ausweitung von internetbasierten Verwaltungsdienstleistungen. Hauptgründe für die Ablehnung des Digitalen Rathauses waren, dass der persönliche Kontakt mit den Sachbearbeitern wichtig oder die augenblicklichen Kontaktmöglichkeiten ausreichend seien (vgl. Abb. 4). 
Abb. 4: Gründe für die Ablehnung des Digitalen Rathauses

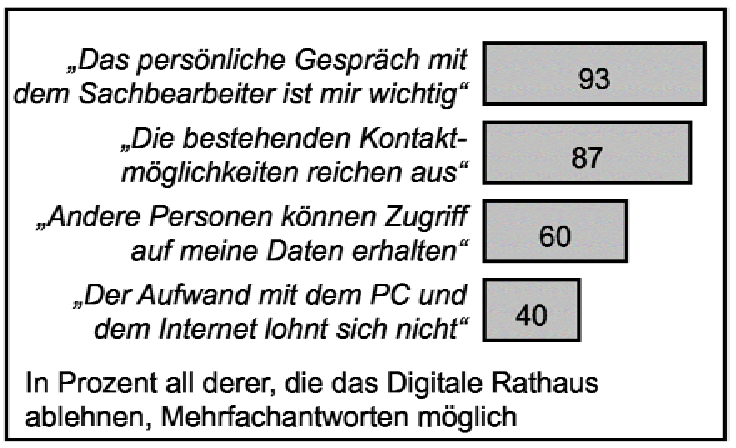

Nach einzelnen potenziellen Online-Verwaltungsangeboten befragt, wünschten sich mehr als die Hälfte derer, die Computer- oder Internetnutzung nicht grundsätzlich ablehnen, die Möglichkeit zur Erledigung aller Verwaltungsangelegenheiten per Internet, die Einrichtung von Beschwerde- oder Kummerkästen, Diskussionsmöglichkeiten mit der Verwaltung und Stadträten oder Informationen über städtische Baumaßnahmen, Verzeichnisse und Handbücher oder Stellenausschreibungen. An der Online-Bekanntmachung von Ausschreibungen von städtischen Aufträgen oder Beschaffungen zeigten noch knapp ein Drittel dieser Gruppe Interesse (vgl. Abb. 5). Da städtische Aufträge nur für einen engeren Kreis der Bürger von direktem Interesse sein dürften, ist dies ein erstaunlich hohes Ergebnis.

Auch eindeutig auf politische Partizipation gezielte Online-Angebote fanden unter den Befragten eine große Zustimmung. $47 \%$ meinten, dass sie an internetbasierten Wahlen teilnehmen würden.

Von den Möglichkeiten, Bürger mittels des Internets in kommunalpolitische Entscheidungsprozesse einzubinden, sind besonders Internetforen interessant. Deswegen wurde gefragt, wer von den Befragten bereit wäre, sich an „Gesprächen im Internet" zu kommunalpolitischen Themen in Form von Chats oder Foren zu beteiligen. $46 \%$ derer, die Computerund Internet nicht grundsätzlich ablehnen, würden sich an solchen Gesprächen beteiligen, $8 \%$ würden sich vielleicht beteiligen. Wären die Bürger persönlich von der zur Diskussion gestellten Thematik betroffen, würden sich $35 \%$ mehr an entsprechenden Angeboten betei- ligen wollen - insgesamt also eine sehr hohe Quote der Beteiligungsbereitschaft. Hauptgründe für die Ablehnung von Internetforen zu kommunalpolitischen Themen waren Zeitmangel $(58 \%)$, mangelndes Interesse (50\%), fehlende Kenntnisse (49\%) und die Ansicht, dass sich dadurch nichts ändere (43\%).

\section{Abb. 5: Erwünschte potenzielle Online- Verwaltungsangebote}

\begin{tabular}{|c|c|}
\hline \multirow{2}{*}{$\begin{array}{r}\text { Beschwerde- oder } \\
\text { Kummerkästen } \\
\text { Vorhaben der Stadt } \\
\text { (z.B. Baumassnahmen) }\end{array}$} & 69 \\
\hline & 63 \\
\hline $\begin{array}{r}\text { Verzeichnisse und } \\
\text { Handbücher }\end{array}$ & 63 \\
\hline $\begin{array}{l}\text { Stellenausschrei- } \\
\text { bungen der Stadt }\end{array}$ & 58 \\
\hline $\begin{array}{l}\text { Diskussionsmöglichkeiten } \\
\text { mit Verwaltung/Stadträten }\end{array}$ & 54 \\
\hline $\begin{array}{l}\text { Ausschreibungen } \\
\text { von Aufträgen }\end{array}$ & 31 \\
\hline Kein Interesse an Angeboten & \\
\hline $\begin{array}{l}\text { Wünsche in \% aller Befragten } \\
\text { Internet nicht grundsätzlich ab } \\
\text { antworten möglich }\end{array}$ & $\begin{array}{l}\text { e Computer oder } \\
\text { Inen, Mehrfach- }\end{array}$ \\
\hline
\end{tabular}

Die Ergebnisse bezüglich möglicher zukünftiger Angebote kontrastieren also die Befunde zur spärlichen Nutzung des Digitalen Rathauses: Die befragten Bürger zeigten sich insgesamt sehr offen für zukünftige Möglichkeiten politischer Partizipation oder für Verwaltungsdienstleistungen via Internet.

\section{Schlussfolgerungen}

Die Internet-Portale der Städte Mannheim und Karlsruhe haben einen hohen Bekanntheitsgrad und werden häufig besucht. Allerdings werden in erster Linie informatorische Angebote genutzt, interaktive und insbesondere transaktive Angebote, die das Herzstück von zukünftigen E-Government-Angeboten bilden sollen, werden überraschend selten in Anspruch genommen.

In Anbetracht der Tatsache, dass die bestehenden Online-Verwaltungsdienstleistungen fast ausschließlich von versierten Internetnutzern (d. h. solchen, die sowohl von zu Hause als auch vom Arbeitsplatz aus Internetzugang haben) in 
Anspruch genommen werden, wird angenommen, dass insbesondere die Vertrautheit mit dem Medium eine Rolle spielt. Voraussetzung für eine stärkere Nutzung wäre also die Verringerung des „Digital Divide“, unter anderem durch die Förderung von Medienkompetenz, wie vielfach gefordert wird.

In Bezug auf internetgestützte Beteiligungsverfahren (E-Democracy) ergibt sich ein spezifisch kommunalpolitisches Problem: Während allgemeines politisches Interesse mit Internetnutzung hinsichtlich sozialstruktureller Kriterien relativ gut konvergiert, ist Interesse an kommunalpolitischen Themen häufig bei denjenigen $\mathrm{zu}$ finden ist, die das Internet seltener nutzen.

Erstaunlich ist jedoch in erster Linie die hohe Diskrepanz zwischen dem Wunsch der Bürger nach Internet-Verwaltungsdiensten sowie der generellen Bereitschaft, diese zu nutzen, und der im Vergleich dazu sehr bescheidenen Inanspruchnahme vorhandener Angebote.

Diese Diskrepanz ist so groß, dass sozialstrukturell unterschiedliche Zugangschancen und Unterschiede in der Medienkompetenz nicht allein als Erklärung hinreichen: Auch von denjenigen, die über Zugang zum Internet verfügen und bei denen eine gute Medienkompetenz vermutet werden darf, nutzen nur ein Bruchteil der Befragten die Angebote des Digitalen Rathauses und dies, obwohl die Existenz des Digitale Rathauses selbst relativ gut bekannt ist. Sicherlich spielt der gegenwärtig geringe Nutzwert der Angebote, der in der Regel als Erklärung für die geringe Nutzung herangezogen wird (vgl. Hoecker 2002), eine wichtige Rolle. Aber auch er kann nur zum Teil als Erklärung für das „Praxis-Defizit“" herangezogen werden. Immerhin hatten alle befragten Bürger in den letzten zwei Jahren Kontakt zu den Ämtern. Trotzdem wurden auch die Möglichkeiten des Digitalen Rathauses, die einen Amtsgang vorbereiten und erleichtern, wie z. B. die Möglichkeiten des Formular-Downloads, erstaunlich selten in Anspruch genommen.

Dennoch sind sozial ungleich verteilte $\mathrm{Zu}-$ gangschancen einerseits und die Frage des eigentlichen Nutzwertes zusammen die Haupterklärungsgründe für die geringe Nutzung des Digitalen Rathauses. Um eine breitere Nutzung von elektronischen Diensten zu erreichen, müsste an beiden Seiten gearbeitet werden.
Die Untersuchung der Fallbeispiele Karlsruhe und Mannheim zeigte zwar konzeptionelle Unterschiede in der Organisierung und den Inhaltsschwerpunkten in den Stadtportalen auf, diese führten aber auf der Ebene des Gebrauchswerts nicht $\mathrm{zu}$ einem nennenswerten Unterschied im letztlich verfügbaren Angebot in den Digitalen Rathäusern, das bei beiden (gerade in der Tiefe) beschränkt bleibt. Dies legt es nahe, dass aktuell wesentliche Voraussetzungen für den Erfolg des Digitalen Rathauses weniger im „Front-Office“, also in der technischen und im weiteren Sinne gestalterischen Neudefinierung der Schnittstelle zu den Bürgern zu finden sind. Entscheidende Hemmnisse scheinen eher in Faktoren zu liegen, die auf verwaltungsinterne Strukturen (Organisierung des Work-Flow im Back-Office: Struktur- und Prozessanpassungen an E-Government-Anforderungen) oder Verantwortlichkeiten außerhalb des kommunalen Einflussbereichs (Anpassungen von Gesetzen und Verwaltungsvorschriften durch Bund und Länder, Koordinierungsinitiativen durch höhere föderale Ebenen, Finanzausstattung der Kommunen) zurückgeführt werden können (siehe hierzu auch Deutscher Städtetag 2002).

Die Erfahrungen der kommunalen Betreiber der Stadtportale weisen ebenso darauf hin, dass E-Government eine Perspektive erfordert, die die beiden Ebenen „Außenbeziehungen der Verwaltung“ und „Inneradministratives“ im Blick behält. Eine intranetbasierte effektive Unterstützung der Abläufe an den Schnittstellen zum Bürger - seien sie elektronisch, im Bürgeramt oder in Call-Centern - und eine Neuordnung inneradminstrativer Abläufe, die medienbruchfreie Arbeitsabläufe ermöglichen, gelten aus dieser Sicht als die wesentlichen Faktoren sowohl für die Umsetzung des Leitbilds der Bürgerorientierung im Sinne einer strikt am Kunden, also den Bürgern, orientierten Bereitstellung der Verwaltungsdienstleistungen, wie auch für die - noch weit entfernte - Erfüllung der Einsparungserwartungen (siehe auch Blumenthal 2002, S. 37). Den hoch gesteckten politischen Zielvorgaben und Erwartungen stehen die Kommunen insofern auch skeptisch gegenüber, da die Bundespolitik spezifische Hemmnisse auf der kommunalen Seite verkenne und ihre Initiativen bisher zu sehr auf rechtliche Rahmensetzungen zur Durchführung von 
Transaktionen (maßgeblich das Signaturgesetz) beschränkt habe.

\section{Ausblick}

In politischen Zielsetzungen wird immer wieder ein möglichst weitgehendes und vollständiges Angebot an Online-Verwaltungsdienstleistungen gefordert: Mit einem größeren Angebot steigt der Nutzwert für den Bürger und somit die Motivation, Zugangshemmnisse $\mathrm{zu}$ überwinden. Mit zunehmender Nutzerzahl wiederum steigen die Rationalisierungseffekte auf der Behördenseite. Ämter und Bürgerbüros werden aber, schon allein aus Gründen der allgemeinen Zugänglichkeit der Verwaltung, zumindest mittelfristig neben dem Digitalen Rathaus Bestand haben. Letztere haben gegenüber OnlineTransaktionen weitere Vorteile. So wird auf dem Amt geholfen, wenn Unklarheiten über notwendige Unterlagen, das Ausfüllen von Formularen oder andere Details der Transaktion bestehen. Bei einem Vorgang, der auf dem Amt angestoßen wird, hat der Bürger einen kompetenten Beamten zur Seite, der bei Bedarf Hilfestellung leistet. Im Großen und Ganzen kann der Bürger also davon ausgehen, dass, wenn er auf das Amt geht und alle nötigen Unterlagen beibringt, der Vorgang abgeschlossen wird.

Bei Online-Verwaltungsdienstleistungen hingegen bestehen diesbezüglich Unsicherheiten. Der Bürger muss weitgehend selbsttätig oder mit lediglich schriftlicher Anleitung den Verwaltungsvorgang bearbeiten. Er muss herausfinden, welche Unterlagen und Formulare er im Einzelnen benötigt und muss in der Lage sein, diese selbständig korrekt auszufüllen. Inwiefern zukünftige Online-Transaktionsprozesse geeignet sein werden, die Nutzer so zu unterstützen, dass diese Unsicherheiten entfallen, bleibt abzuwarten. Der persönliche Kontakt zum Sachbearbeiter hat jedoch zumindest zurzeit eine Qualität, die online nicht ohne weiteres substituierbar ist. Dafür spricht auch, dass der Hauptablehnungsgrund für das Digitale Rathaus darin besteht, dass der persönliche Kontakt zum Sachbearbeiter ,ausreiche“ (vgl. Abb. 4).

Bei Online-Verwaltungsdienstleistungen entstehen also zusätzliche Unsicherheiten. Diese Unsicherheiten sind individuell unterschiedlich und hängen damit zusammen, wie sehr der
Bürger mit Verwaltungsakten vertraut ist und die spezifische Verwaltungslogik nachvollziehen kann. Man könnte hier von einer „Behördenkompetenz" sprechen, die neben den anderen Voraussetzungen gegeben sein muss, um den Mehrwert von Online-Verwaltungsdiensten nutzen zu können.

Wenn Online-Verwaltungsdienstleistungen in dieser Art als Alternative zum Gang ins Bürgerbüro gedacht werden, sind möglicherweise bislang weniger beachtete Aspekte zur Erklärung der Diskrepanz zwischen der generellen Bereitschaft, Verwaltungsdienstleistungen per Internet in Anspruch zu nehmen, und der tatsächlichen Nutzung heranzuziehen. Eine Betrachtung von Bürger-Behörden-Transaktionen als soziale Interaktion kann hier vielleicht neue Aufschlüsse liefern.

\section{Anmerkungen}

1) Gefördert von „Innovations- und Technikanaly$\mathrm{se}^{\text {" des }}$ Bundesministeriums für Bildung und Forschung (BMBF) als Teil des Vorhabens „Technologie, Kommunikation und Diskurs im medialen Zeitalter"; Förderkennzeichen 16/1477.

2) Die Verwendung des Begriffs „Digitales Rathaus" ist dabei keineswegs einheitlich - weder im Gebrauch auf den Portalen selbst noch in den Diskussionen über elektronische Verwaltungsdienste. Im Folgenden ist, wenn vom Digitalen Rathaus die Rede ist, der Bereich auf den Portalen gemeint, in dem die Bürger mit der Verwaltung zur Vorbereitung oder Abwicklung von Dienstleistungen interaktiv oder transaktiv in Kontakt treten können. Alternativ zur Bezeichnung „Digitales Rathaus“ werden vielfach auch Begriffe wie ,virtuelles Rathaus“ oder ,,elektronisches Bürgeramt“ benutzt.

3) In den einzelnen Kommunen wurden dabei jeweils eigene Wege gegangen; insbesondere wurden je eigene Schwerpunkte im Ausbau gesetzt. Vgl. zu Periodisierungsversuchen und zur Bestimmung verschiedener "Generationen“ kommunaler Internetauftritte bzw. Digitaler Rathäuser: Blumenthal 2002, S. 37 f.; StapelSchulz, Eifert, Siegfried 2002, S. 5; Initiative D21 2002a, S. 6.

4) Siehe hierzu auch den Aufsatz von Behringer in diesem Schwerpunkt. Während bei Behringer grundsätzliche Fragen der Konzeption, Organisation und Positionierung kommunaler Portale (und von Karlsruhe.de im Besonderen) im Vordergrund stehen, konzentriert sich der vorlie 
gende Beitrag im Wesentlichen auf die Digitalen Rathäuser auf Karlsruhe.de und Mannheim.de als „Orte“ der Bereitstellung von Verwaltungsdiensten im Internet.

5) Die Nutzerführung über Lebenslagen soll dem Bürger verschiedene Angebote, die mit seiner aktuellen Lebenssituation in Verbindung stehen, gebündelt präsentieren. So umfasst in der lediglich in Ansätzen entwickelten - Mannheimer Ausgestaltung die Lebenslage „Heirat“ z. B. als städtische Anlaufstellen Links zu den Bürgerdiensten und Standesämtern und verweist darüber hinaus auf Frisöre, Juweliere, Partyservices usw.

6) Vgl. zu unterschiedlichen Organisationsmodellen für den Betrieb von Stadtportalen StapelSchulz, Eifert, Siegfried 2002, speziell zu Karlsruhe und Mannheim: S. 26 u. S. 29. Siehe zu Mannheim.de auch Blumenthal 2001.

7) Diese Darstellung bezieht sich auf den Stand der Internetseiten zur Zeit der Telefonbefragung (Frühjahr 2002). Mittlerweile sind jeweils neue Versionen der Stadtportale im Netz.

8) Auf beiden Stadtportalen befinden sich auch alphabetische Listen mit den städtischen und stadtnahen Dienstleistungsangeboten; die Suche über eine Suchmaschine wird ebenfalls von beiden Städten angeboten.

9) Vgl. z. B. von Lucke und Reinermann 2002, Initiative D21 2002a, Bertelsmann Stiftung 2002, Aichholzer und Schmutzer 1999, Accenture 2002, CGEY 2001.

10) Wenn auch inneradministrative Prozesse mit betrachtet werden, bietet es sich an, die einzelnen Stufen weiter zu differenzieren und z. B. danach zu unterscheiden, inwiefern die Anwendungen Umstellungen in den Verwaltungen erfordern, oder ob ein Vorgang auch innerhalb der Verwaltung medienbruchfrei, d. h. (automatisiert) in elektronischer Form in den WorkFlow eingeht. Vgl. zu einem solchen Modell das nach sechs Stufen unterscheidende Projekt „Key Elements for electronic Local Authorities' Networks“ (KEeLAN) (Wenzel 2002), siehe auch http://www.keelan.elanet.org.

11) Von E-Government im engeren Sinn wird häufig erst gesprochen, wenn die Digitalen Rathäuser Transaktionen bieten. In einer strengeren Fassung des Transaktionsbegriffs wird zudem vorausgesetzt, dass auch in der Verwaltung selbst die Abwicklung des Vorgangs medienbruchfrei erfolgt.

12) Mithin können auch interaktive Angebote, für die Schriftformbindung, aber keine persönliche Unterschrift vor Ort vorgeschrieben ist, Ämtergänge ersparen. Dasselbe gilt allerdings auch für die telefonische Bestellung des Formulars und den anschließenden Versand auf dem Postweg.

13) Inwiefern Anregungen aus dem Forum tatsächlich in die ,realen“ Arbeitsgruppen des Partizipationsverfahrens eingeflossen sind, kann hier nicht geprüft werden. Bis kurz vor Abschluss des Verfahrens wurde www.city2015.de von der die gesamte Bürgerbeteiligung moderierenden „Kommunalentwicklung BW“ betreut. Mit Übernahme der Seiten durch das Presse- und Informationsamt der Stadt Karlsruhe wurden diese umgestaltet und dienten lediglich noch als - umstrittene, weil vielfach als einseitig empfundene - Informationsbasis. Im Zuge dieser Umgestaltung wurde das interaktive Element der Seiten, das Forum, eingestellt.

14) Mit Blick auf die Reorganisation der Binnenstruktur und Prozessneuordnung durch E-Government kann aber festgehalten werden, dass sich Mannheim in der Gestaltung seiner Schnittstelle zu den Bürgern (und Unternehmen) stärker von der bestehenden Verwaltungsarchitektur löst.

15) Diese Stichprobe stimmt in der Verteilung über sozialstrukturelle Variablen gut mit den Statistiken von Karlsruhe und Mannheim überein, lediglich Ausländer sind leicht unterrepräsentiert.

16) "The population of Internet users is strongly biased along certain socio-demographic variables like age, gender, education and income. This is termed a division between information haves and have-nots, users and losers or as a digital divide" (Welling und Kubicek 2000, S. 1).

\section{Literatur}

Accenture, 2002: Visionen mit Pragmatismus: eGovernment in Deutschland 2002; http://www. accenture.de/index $2 . h t m l$ ?/static_common/egov_ form.jsp

Aichholzer, G.; Schmutzer, R., 1999: E-Government. Elektronische Informationsdienste auf Bundesebene in Österreich. Studie im Auftrag des Bundeskanzleramts. Wien: Institut für Technikfolgen-Abschätzung der Österreichischen Akademie der Wissenschaften

Bertelsmann Stiftung, 2002: Balanced E-Government. Elektronisches Regieren zwischen administrativer Effizienz und bürgernaher Demokratie. Gütersloh; http://www.begix.de/studie/download.html

Blumenthal, J., 2001: Erfahrungen mit Bürgerservices/Portalen. In: Bundesministerium für Wirtschaft und Technologie (Hrsg.): Bürgerkommune im Netz. Tagungsband zum 2. Media@Komm-Fachkongress am 11./12.6.2001 in Esslingen. Berlin, S. 230 - 234; http://www.mediakomm.net/documents/kongress/ esslingen/doku500a.pdf 
Blumenthal, J., 2002: Die Rolle Digitaler Rathäuser beim E-Government. In: Verwaltung und Management, 8. Jg., Nr. 1, S. $37-40$

CGEY (Cap Gemini Ernst \& Young), 2001: Webbasierte Untersuchung des elektronischen ServiceAngebots der öffentlichen Hand; http://www.de.cgey. com/servlet/PB/show/1002171/e-government.pdf

Deutscher Städtetag, 2002: eGovernment ist der Schlüssel zur modernen Verwaltung. In: Der Städtetag, 55. Jg., Nr. 7/8, S. $52-54$

Hoecker, B., 2002: Mehr Demokratie via Internet? Die Potenziale der digitalen Technik auf dem empirischen Prüfstand. In: Aus Politik und Zeitgeschehen, 52. Jg., Nr. 39/40, S. 37-45

Initiative D21 (Hrsg.), 2002a: E-Town 2002. Deutschlands digitale Hauptstädte. Berlin; http://www.initiatived21.de/projekte/ publikationen/etown2002.pdf

Initiative D21 (Hrsg.), 2002b: (N)Onliner Atlas 2002. Eine Topographie des digitalen Grabens durch Deutschland. Bielefeld, Berlin

Kubicek, H.; Wind, M., 2002: eGovernment ist mehr als Formulare zum Herunterladen. Das 24-StundenRathaus. In: Der Städtetag, 55. Jg., Nr. 6, S. 11 - 14

Lenk, K., 2002: Elektronische Bürgerdienste im Flächenland als staatlich-kommunale Gemeinschaftsaufgabe. In: Verwaltung und Management, 8. Jg., Nr. 1, S. $4-10$

Lucke, J. von; Reinermann, H., 2002: Speyerer Definition von Electronic Government. In: H. Reinermann; Lucke, J. von (Hrsg.): Electronic Government in Deutschland. Ziele - Stand - Barrieren - Beispiele - Umsetzung. (Speyerer Forschungsberichte; Bd. 226). Speyer: Forschungsinstitut für öffentliche Verwaltung, S. $1-8$

Stapel-Schulz, C.; Eifert, M.; Siegfried, C., 2002: Organisations- und Kooperationstypen kommunaler Internetauftritte (Arbeitspapiere aus der Begleitforschung zum Städtewettbewerb Media@Komm). Berlin; http://www.mediakomm.net/ documents/forschung/mk-bf---arbeitspapier-6.pdf

Welling, S.; Kubicek, H., 2000: Measuring and bridging the Digital Divide in Germany. Bremen

Wenzel, W., 2002: The KEeLAN-Report. Conference Presentation; http://www.keelan.elanet.org/ egovernment/documentlibrary.asp?taskhidden= showdocument\&documentid $=20$

\section{Kontakt}

Forschungszentrum Karlsruhe GmbH

Institut für Technikfolgenabschätzung und Systemanalyse (ITAS)

Postfach 36 40, 76021 Karlsruhe

Fax: +49 (0) 7247 / 82 - 6045

Internet: http://www.itas.fzk.de

Matthias Werner, M.A.

Tel.: +49 (0) 7247 / 82 - 6397

E-Mail: werner@itas.fzk.de

Martin Bechmann, Dipl.-Soz.

Tel.: +49 (0) 7247 / $82-6875$

E-Mail: martin.bechmann@itas.fzk.de

\section{$《 \Downarrow$}

